\title{
Mucosal immunology in low- and middle-income countries
}

\section{P Garside ${ }^{1}$}

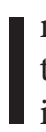
$\mathrm{n}$ this issue, several articles address the importance and study of mucosal immunology in low- and middleincome countries. The reviews were contributed by scientists working in the field, and we have commentaries from two of the major organizations that fund research in these areas.

The latest developments in the mucosal immunology of viral, bacterial, and helminth infections are discussed, as is their direct impact on human health. The risk factors for these infections and potential strategies for their prevention and treatment are also highlighted. Overall, the articles address a number of common problems and themes. In scientific and clinical terms, the important concept of multiple insults and parameters impacting mucosal immunity and therefore being risk factors for, among others, bacterial translocation and bacterial infection has been raised. However, also of note is the importance of undertaking studies in the human populations in which the science will be applied, the difficulties of carrying out such work, and the potential impact of public health interventions. As such, prioritizing funding will be a difficult and crucial task. In relation to this, we are delighted to have commentaries from two of the major funding bodies in the area.

One commentary, a report of a recent Gates Foundation-sponsored meeting, addresses the importance of, and challenges facing, mucosal immunology in developing countries and provides insight into what the problems and priorities might be. The other commentary was contributed by Wellcome Trust staff members who are involved in science funding in low- and middle-income countries and focuses on regulatory and policy issues as well as current priorities for health research and infrastructure.

We hope you will enjoy these articles and perhaps reflect on the fact that an enhanced understanding of mucosal immunology could have a major and rapid impact on the lives of millions of people in the most populated and rapidly developing and expanding areas of the world.

(C) 2009 Society for Mucosal Immunology 